\title{
DNA and LC-MS/MS Finger printings As Novel Tools for the Standardization of Crude Herbal Drugs
}

\section{Pankaj Gupta*}

School of Medical \& Allied Sciences, K.R. Mangalam University, Sohna Road, Gurgaon, Haryana, India.

\section{Editorial}

Standardization of crude herbal drugs refers to analysing the quality and purity of the crude drugs before their further processing and remains a prerequisite step for the preparations of herbal formulations. It involves authentication of crude drugs, analysing the quality and purity of the crude drugs via several means of evaluation such as organoleptic, microscopic, chemical, physical and biological means of evaluation. Among these evaluation techniques used for standardization of crude drugs, there are various methods that have been adopted since time such as morphological and sensory evaluation, chemical tests that have used for preliminary phytochemical screening, determination of ash value, extractive value, foreign matter etc. However, today's era involves the standardization of crude drugs using advanced analytical techniques such as high performance liquid chromatography (HPLC), gas chromatography (GC) and mass spectroscopy (MS) in addition to the previously used techniques. The most recent methodologies engage very sophisticated analytical instruments such as LC-MS/MS (liquid chromatography tandem mass spectroscopy) and PCR (polymerase chain reaction) - based methods for the standardization purposes.
Among the newer methodologies, DNA fingerprinting is one such technique which has evolved with time and has been found to possess tremendous potential for the standardization of crude drugs. In this technique, the DNA analysis of the crude drug has been used for its authentication and for the determination of its quality and purity. This technique also finds application in differentiating the herbal drug from various other adulterants or substituted drugs and offers unique advantage as the DNA fingerprint genome for any crude drug remains same irrespective of the part of the plant that have been used for extraction. Another technique which finds tremendous application is LC-MS/MS. This technique not only provides information regarding the molecular weight of all the parent compounds that are present in the crude drugs but also provides information regarding the daughter nuclei present via predicting the fragmentation patterns of the parent compounds. This technique offers advantage in that the daughter nuclei can be predicted from the parent nucleus that would help in differentiating two compounds with the same molecular weights and determination of fragmentation patterns that would differentiate the herbal drug from various other adulterants. Thus, the problem of quality assurance of herbal drugs can at large be solved with the use of DNA fingerprinting and LC-MS/MS fingerprinting techniques.
*Corresponding author: Gupta P, Assistant Professor, School of Medical \& Allied Sciences, K.R. Mangalam University, Sohna Road, Gurgaon-122103, Haryana, India, Tel: +91-11-9818256122; E-mail: gupta.aiims@gmail.com

Received May 25, 2015; Accepted May 26, 2015; Published May 30, 2015

Citation: Gupta P (2015) DNA and LC-MS/MS Finger printings As Novel Tools for the Standardization of Crude Herbal Drugs. Nat Prod Chem Res 3: e112. doi:10.4172/2329-6836.1000e112

Copyright: (c) 2015 Gupta P, This is an open-access article distributed under the terms of the Creative Commons Attribution License, which permits unrestricted use, distribution, and reproduction in any medium, provided the original author and source are credited. 